\title{
Comparison of koala LPCoLN and human strains of Chlamydia pneumoniae highlights extended genetic diversity in the species
}

\author{
Candice M Mitchell ${ }^{1}$, Kelley M Hovis ${ }^{2}$, Patrik M Bavoil ${ }^{2}$, Garry SA Myers ${ }^{3}$, Jose A Carrasco ${ }^{2}$, Peter Timms ${ }^{*}$
}

\begin{abstract}
Background: Chlamydia pneumoniae is a widespread pathogen causing upper and lower respiratory tract infections in addition to a range of other diseases in humans and animals. Previous whole genome analyses have focused on four essentially clonal (> 99\% identity) C. pneumoniae human genomes (AR39, CWL029, J138 and TW183), providing relatively little insight into strain diversity and evolution of this species.

Results: We performed individual gene-by-gene comparisons of the recently sequenced C. pneumoniae koala genome and four C. pneumoniae human genomes to identify species-specific genes, and more importantly, to gain an insight into the genetic diversity and evolution of the species. We selected genes dispersed throughout the chromosome, representing genes that were specific to C. pneumoniae, genes with a demonstrated role in chlamydial biology and/or pathogenicity $(n=49)$, genes encoding nucleotide salvage or amino acid biosynthesis proteins $(n=6)$, and extrachromosomal elements (9 plasmid and 2 bacteriophage genes).

Conclusions: We have identified strain-specific differences and targets for detection of $C$. pneumoniae isolates from both human and animal origin. Such characterisation is necessary for an improved understanding of disease transmission and intervention.
\end{abstract}

\section{Background}

The Chlamydiaceae are obligate intracellular pathogens that undergo a unique biphasic developmental cycle involving the inter-conversion between the extracellular infectious elementary body and the intracellular, replicative reticulate body. Chlamydia 'Chlamydophila' pneumoniae is probably one of the most successful chlamydial species, having established a niche in a range of warm-blooded (homoeothermic) and cold-blooded (poikilothermic) hosts, including humans, horses, marsupials, frogs and reptiles [1-5]. C. pneumoniae human infections are associated with bronchitis, pharyngitis, community-acquired pneumonia and more recently chronic diseases, such as atherosclerosis and stroke [6,7] myocarditis [8], multiple sclerosis [9] and Alzheimer's disease [10].

\footnotetext{
* Correspondence: p.timms@qut.edu.au

${ }^{1}$ Institute of Health and Biomedical Innovation, Faculty of Science and Technology, Queensland University of Technology, Kelvin Grove, Queensland, 4059, Australia
}

C 2010 Mitchell et al; licensee BioMed Central Ltd. This is an Open Access article distributed under the terms of the Creative Commons Attribution License (http://creativecommons.org/licenses/by/2.0), which permits unrestricted use, distribution, and reproduction in any medium, provided the original work is properly cited.
Australia's native icon, the koala (Phascolarctos cinereus), is found throughout Australia's north-eastern and southern eucalypt regions and is commonly infected with chlamydiae [11-14]. While the decline in koala populations has largely been the result of hunting and a diminished habitat, there is great concern for the koala due to an increased incidence of disease [15]. It is estimated that almost all of the nations free-range koala populations and many in captive populations are affected by C. pneumoniae and/or C. pecorum (the most common of the two species). C. pneumoniae-infected koalas may develop a respiratory illness similar to that in humans with clinical signs of sneezing, coughing, nasal discharge and chest congestion [16,17]. C. pneumoniae koala is not restricted to the respiratory tract and has been isolated from ocular and urogenital tract sites (often in conjunction with C. pecorum), although disease at these sites is poorly understood [13].

Exposure to C. pneumoniae is widespread due to effective aerosol transmission and outbreaks have been reported in humans [18-21], horses [22], frogs [23] and 
koalas [13]. However, the original source of infection remains undetermined. Myers et al. [24] recently published the full $1.24 \mathrm{Mbp}$ genome sequence of the C. pneumoniae koala LPCoLN isolate, the first analysis of a C. pneumoniae genome from a non-human host species. This study revealed that the five C. pneumoniae genomes were highly similar in genomic organisation and gene order, although some notable differences were observed [24]. In contrast to the highly conserved human-derived isolates, a relatively high number of single nucleotide polymorphisms, SNPs (6213) differentiated koala LPCoLN from human AR39 [24]. In the proposed phylogeny (based on SNPs from 111 highly conserved genes), which encompassed all five C. pneumoniae genomes and five sequenced animal chlamydial genomes (C. pecorum E58, C. muridarum Nigg, C. caviae GPIC, C. psittaci $6 \mathrm{BC}$ and C. abortus s26/3), koala LPCoLN was basal to the C. pneumoniae human isolates (larger genome and many full-length genes relative to human isolates).

In the present report we perform a comparative analysis of the whole koala LPCoLN genome sequence, highlighting the components that distinguish the strain isolated from the koala (animal), from those isolated from humans. These comparisons point to candidates of strain-specific adaptations and may provide potential targets for improved diagnostic tests, therapeutic intervention and epidemiological investigations.

\section{Results}

We used a comparative genomics approach to identify genetic characteristics that were either unique to C. pneumoniae or were commonly shared with chlamydial species and other organisms. Overall, we analysed a total of 66 genes for key similarities and differences between human and animal strains of C. pneumoniae (see Additional file 1 for list of 66 genes analysed). We chose to use our C. pneumoniae animal genome as the reference genome and compared the available human genomes to it.

\section{Chlamydia pneumoniae-specific genes}

Using tblastx and tblastn, we searched other genomes for orthologs of the genes identified from individual gene-by-gene comparisons of the five C. pneumoniae genomes. The comparative approach identified 140 genes that were specific to C. pneumoniae and for which no significant similarity was detected in any other organism (Additional file 2). Many of these speciesspecific genes are short open reading frames (ORFs) that have been annotated as genes. The large number of short hypothetical ORFs makes it difficult to determine whether these genes are 'real' or artefacts of the genome annotation or sequencing process. Until these proteins are systematically studied in the future, it cannot be determined whether these proteins are valid or too short to be protein-coding genes. Genes with suggested or predicted functions include putative lipoproteins and chlamydial inclusion membrane proteins (IncA). Several hypothetical proteins are clustered together (including CPK_ORF00340-343, 389-401, 496-498, 567-569, 658-661, 969-980: LPCoLN locus designation, CPK), suggesting that they may exist in an operon and might be functionally related.

\section{Genes with a demonstrated role in chlamydial biology and/or pathogenicity}

One of the most striking differences between the C. pneumoniae koala and human genomes were changes associated with the polymorphic membrane proteins (Pmps). Like C. pneumoniae of human origin [25,26], koala LPCoLN is predicted to encode 21 Pmps that are phylogenetically related to one of six basic subtypes (pmp A, B/C, D, E/F, G/I and $\mathrm{H}$; Additional files 3 and 4) [25,27-29]. The organisation of the pmp loci of koala LPCoLN is conserved relative to the C. pneumoniae human isolates. However, where the four human isolates carry several interrupted pmp genes (Additional file 3), koala LPCoLN carries uninterrupted, full-length versions of the same genes, including pmpG3, pmpG4 and $p m p$ E3 (Additional file 3). A global comparison of all pmp sequences reveals a total of 2015 SNPs (of which 994 generated an amino acid change; see Additional file 5 for approximate SNP positions) differentiating koala LPCoLN from the four C. pneumoniae human isolates, with the highest percentage of SNPs observed in $p m p \mathrm{E} 4$ $(30.46 \%)$ and $p m p \mathrm{E} 3$ (6.95\%). In addition to SNPs, several strain-specific (human versus animal) indels (insertions and deletions) were evident in $p m p \mathrm{~B}, p m p \mathrm{E} 1$, pmp $\mathrm{G} 2, p m p \mathrm{G} 4, p m p \mathrm{G} 5, p m p \mathrm{G} 7, p m p \mathrm{G} 10$, and pmp G13 (Additional file 3). Interestingly, the pmpG5 pseudogene is interrupted by a stop codon in all five strains albeit at different sites: LPCoLN carries a seven nt indel (GAT GTA C) at nt position 332, resulting in a TAA stop codon at nt position 346 , while the four human isolates have a SNP (C to T) at nt 1483, resulting in a TAA stop codon (data not shown). Previous analyses of human isolates have revealed variable numbers of $393 \mathrm{nt}$ tandem repeat segments in pmpG6, including two repeats in AR39 [25] and J138 [30], and three repeats in TW183 (Geng MM, Schuhmacher A, Muehldorfer I, Bensch KW, Schaefer KP, Schneider S, Pohl T, Essig A, Marre R, Melchers K: The genome sequence of Chlamydia pneumoniae TW183 and comparison with other Chlamydia strains based on whole genome sequence analysis, submitted) and CWL029 [26]. The LPCoLN genome carries three variable tandem repeats in $p m p \mathrm{G} 6$. 
Type III secretion (T3S) occurs independently of the sec pathway and requires assembly of a secretion apparatus composed of approximately 20 proteins. However, in this analysis we looked at more than just the apparatus proteins; we also examined potential secreted proteins and chaperone proteins involved in T3S. Additional file 6 compares 26 proteins from LPCoLN to putative orthologs of other chlamydial species. Ten apparatus-encoding genes (CDSs CPK_ORF00106, 111, $115,231,232,233,234,236,830$ and 831), which were either annotated as such and/or found to be homologous to previously studied proteins in other chlamydial spp. were examined. Genetic comparisons indicate $\geq$ 98.2\% nucleotide sequence identity between each koala LPCoLN T3S apparatus gene and orthologs from the human isolates. Similarly, comparisons of genes which were annotated as, or are similar to, chaperone-encoding genes that assist in the folding of effectors demonstrated high conservation with $\geq 99.3 \%$ sequence identity to the equivalent genes in the C. pneumoniae isolates from humans.

Nine putative effector-encoding genes (CDSs CPK_ORF00107, 216, 217, 430, 445, 446, 799, 800 and 832) of C. pneumoniae koala LPCoLN were compared to their counterparts in the human isolates. As with the T3S apparatus proteins, all koala LPCoLN effectors exhibited $\geq 98.2 \%$ sequence identity with their human isolate counterparts (Additional file 6).

The plasticity zone or replication termination region is a hypervariable region that is linked to genetic differences in chlamydial pathogen-host relationships. The membrane attack complex/perforin (MACPF) of the plasticity zone is one such protein that showed varied degrees of polymorphism among the chlamydial species. There was a significant length polymorphism differentiating the MACPF of C. pneumoniae koala (2457 nt CPK_ORF00685) from all four human isolates which encode a predicted defective MACPF separately incorporated into two ORFs, CP_0594 (381 nt) and CP_0593
(1236 nt) (Figure 1). A comparison with other chlamydial species showed that a C. pneumoniae ancestor separated from other 'Chlamydophila spp.' before the large indel was removed from the human isolates. Similar to the C. pneumoniae koala LPCoLN MACPF, C. trachomatis serovar, A/HAR, B/Jali20/OT, D/UQ-3/CX, L2b/ $\mathrm{UCH}-1 /$ proctititis, $\mathrm{L} 2 / 434 / \mathrm{Bu}$ and $C$. muridarum Nigg isolates had a full-length version (Additional file 7), while variations of the MACPF were observed in C. abortus S26/3, C. caviae GPIC and C. felis Fe/C-56 isolates (Additional file 7), which showed frame disruptions (likely pseudogenes). Protochlamydia amoebophila UWE25 did not have any detectable MACPF orthologs. MotifScans of the chlamydial MACPF (with the exception of C. caviae, C. abortus and C. felis) revealed an MIR (Mannosyltransferase, Inositol 1,4,5-trisphosphate receptor and Ryanodine receptor) motif, suggestive of a possible ligand transferase function. The size variation of the C. pneumoniae MACPF may serve as a useful marker in future genetic investigation. For example, the MACPF gene sequence may potentially differentiate C. pneumoniae animal isolates from C. pneumoniae human isolates.

\section{Genes involved in nucleotide salvaging pathways or amino acid biosynthesis}

All sequenced chlamydial genomes to date encode a CTP synthetase, the enzyme that converts UTP to CTP, and an ATP/ADP translocase [25,31]. However, comparative genomic analysis suggests that multiple modifications have occurred in nucleotide salvage pathways during the course of chlamydial evolution, as revealed by the variable presence of $u d k$ (uridine kinase), $p y r E$ (pyrimidine phosphoribosyl transferase), guaB (IMP dehydrogenase), guaA (GMP synthase) and add (adenosine deaminase) in different isolates (Table 1).

C. pneumoniae is the only chlamydial species to have a $u d k$ gene for UMP production. Our examination of the C. pneumoniae sequence revealed that the 3' end of

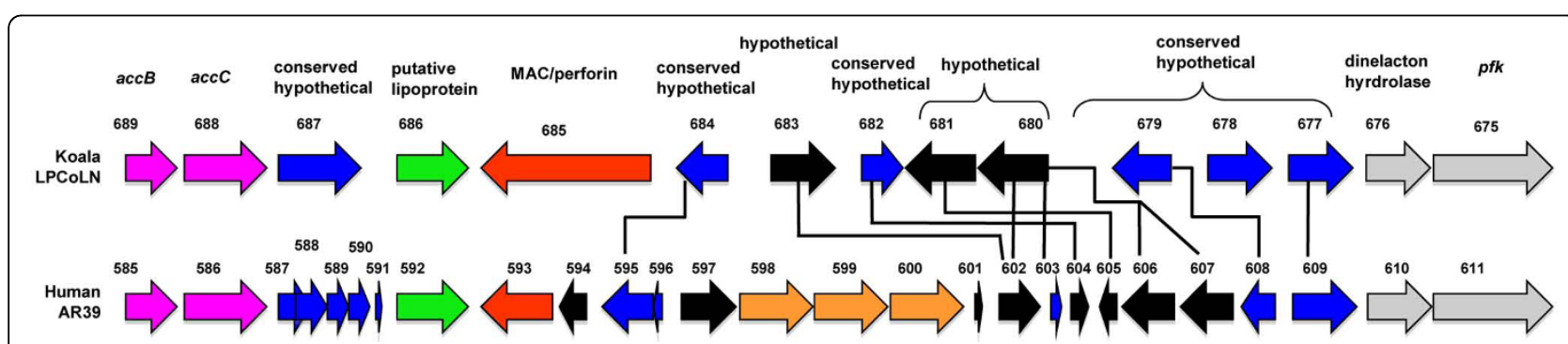

Figure 1 Organisation of the $\boldsymbol{C}$. pneumoniae plasticity zone. A comparison of the C. pneumoniae koala LPCoLN and human AR39 genomes revealed evidence of fragmentation, gene decay, gene gain/loss in the plasticity zone. Genes are labelled with the published locus numbers. Lines connect orthologs. Role categories and colours are as follows: fatty acid and phospholipid metabolism, magenta; conserved hypothetical proteins, blue; cell envelope, light green; hypothetical proteins, black; biosynthesis of purines, pyrimidines, nucleosides, and nucleotides, orange; energy metabolism, light gray. Arrows indicate the direction of transcription. 
Table 1 Chlamydiaceae genome features with suspected host and niche specific genes

\begin{tabular}{|c|c|c|c|c|c|c|c|c|}
\hline Species & $\begin{array}{l}\text { Genome } \\
\text { size (nt) }\end{array}$ & $\begin{array}{l}\text { Protein coding } \\
\text { sequences }\end{array}$ & $\begin{array}{l}\text { Tryptophan } \\
\text { metabolism }\end{array}$ & $\begin{array}{l}\text { Toxin } \\
\text { genes }\end{array}$ & Plasmid & Bacteriophage & $\begin{array}{l}\text { tyrP } \\
\text { copies }\end{array}$ & $\begin{array}{c}\text { Nucleotide } \\
\text { salvaging }\end{array}$ \\
\hline $\begin{array}{l}\text { C. pneumoniae } \\
\text { AR39 }\end{array}$ & 1229853 & 1052 & tph & Absent & Absent & Present & Two & guaBA-add, udk, pyrE \\
\hline $\begin{array}{l}\text { C. pneumoniae } \\
\text { CWL029 }\end{array}$ & 1230230 & 1073 & tph & Absent & Absent & Absent & Two & guaBA-add, udk, pyrE \\
\hline $\begin{array}{l}\text { C. pneumoniae } \\
\text { J138 }\end{array}$ & 1226565 & 1072 & tph & Absent & Absent & Absent & One & guaBA-add, udk, pyrE \\
\hline $\begin{array}{l}\text { C. pneumoniae } \\
\text { TW183 }\end{array}$ & 1225935 & 1113 & tph & Absent & Absent & Absent & One & guaBA-add, udk, pyrE \\
\hline $\begin{array}{l}\text { C. pneumoniae } \\
\text { LPCoLN }\end{array}$ & 1241024 & 1095 & tph & Absent & Present & Present* & One & $u d k, p y r E$ \\
\hline C. felis Fe/C-56 & 1166239 & 1005 & $\operatorname{trp} A B F C D R, k y n U$ & Absent & Present & Absent & One & guaBA-add, pyrE \\
\hline C. caviae GPIC & 1173390 & 1009 & $\begin{array}{l}\operatorname{trp} A B F C D R, k y n U, \\
\text { prsA, tph }\end{array}$ & Present & Present & Present & One & guaBA-add, pyrE \\
\hline C. abortus $\$ 26 / 3$ & 1144377 & 961 & tph & Absent & Absent & Present & One & $\begin{array}{c}\text { guab (pseudogene), } \\
\text { pyrE }\end{array}$ \\
\hline $\begin{array}{l}\text { C. muridarum } \\
\text { Nigg }\end{array}$ & 1069412 & 924 & none & Present & Present & Absent & Two & guaBA-add, upp \\
\hline $\begin{array}{l}\text { C. trachomatis } \\
\text { serovar D }\end{array}$ & 1042519 & 894 & $\operatorname{trp} A B C R$ & Present & Present & Absent & Two & None of the above \\
\hline
\end{tabular}

tph, tryptophan hydroxylase; trp, tryptophan biosynthesis; kynU, kynureninase; prs $A$, ribose-phosphate pyrophosphokinase; gua, purine biosynthesis; add, adenosine deaminase; $u d k$, uridine kinase; $p y r E$, UMP synthase; upp, uracil phosphororibosyl transferase. * Asterisk indicates remnants.

the gene was unique to the species. No significant sequence similarity was observed in other organisms between nt regions 541-669, indicating that this region might be specific for $C$. pneumoniae. A sequence alignment of the full-length $u d k$ gene identified only three SNPs (one amino acid change) differentiating koala LPCoLN from the sequenced human isolates.

The bacterial pyrimidine biosynthesis pathway includes several enzymes for the conversion of UMP into CTP. However, all chlamydial genomes thus far lack the genes for most of the pathway with the exception of the last few steps (Additional file 8). C. pneumoniae of koala and human origins, C. felis, C. caviae and $C$. abortus have the pyrE gene, encoding an orotate phosphoribosyl transferase involved in pyrimidine biosynthesis. However, the final step in de novo pyrimidine biosynthesis is via orotidine-5'-monophosphate decarboxylase $(p y r F)$ and this gene is missing from all chlamydial genomes.

The purine biosynthesis pathway is also incomplete, similar to the pyrimidine pathway, with many genes variably missing in the chlamydial genomes (Table 1 ). The only four genes that are absent from the koala LPCoLN genome but are present in the human genomes include CP_0597, which encodes a hypothetical protein, guaB (IMP dehydrogenase), guaA (GMP synthase) and add (AMP adenosine deaminase), which are involved in purine ribonucleotide biosynthesis. The guaA and add sequences of the $C$. pneumoniae human isolates were identical, while guaB fragmentation was evident in TW183, CWL029 and J138 isolates with a deleted
$324 \mathrm{nt}$ at the 5 ' end of the sequence (Figure 1). The CP_0597 gene resides next to this guaBA-add cluster (Figure 1), which may indicate that this gene may also be involved in purine biosynthesis.

The tryptophan biosynthesis operon is missing from several chlamydial species including $C$. muridarum Nigg, C. abortus S26/3, and C. pneumoniae of both koala and human origin (Table 1). Despite this absence, C. pneumoniae koala and human encode a functional aromatic amino acid (tryptophan) hydroxylase, although the koala LPCoLN isolate is missing the extended $\mathrm{N}$-terminal region [32]. While variations in C. pneumoniae tyrP (tryptophan tyrosine permease) copy numbers have been found between human isolates [33], we report that koala LPCoLN, a respiratory isolate, has a single copy of tyrP. A comparison of the tyrP sequence from all five sequenced (full-genome) C. pneumoniae isolates revealed that the sequence was highly conserved across the seven copies of tyrP, revealing only nine SNPs including seven unique to koala LPCoLN, four of which led to an amino acid change. Previously, Gieffers et al. [33] published a tyrP-specific SNP profile of 20 C. pneumoniae human isolates, and here we report the SNP profile of two additional respiratory isolates J138 (CGGGG) and LPCoLN (CAAGG).

\section{Extrachromosomal elements of the Chlamydiaceae}

Extrachromosomal plasmid sequences pCpnKo from C. pneumoniae koala LPCoLN [24], pCpnE1 from C. pneumoniae horse N16 [34], pCpA1 from C. psittaci avian N352 (Lusher ME, Gregory J, Storey CC, 
Richmond SJ: Analysis of the complete nucleotide sequence of the plasmid pCpA1 isolated from an avian strain of Chlamydia psittaci, Submitted), pCfe1 from $C$. felis feline $\mathrm{Fe} / \mathrm{C}-56$ [35], pCpGP1 from C. caviae guinea pig GPIC [36], pMoPn from C. muridarum mouse Nigg [25], and pCTA, pJALI, pSW2 and pLVG440 from $C$. trachomatis human serovars A [37], B [38], E [38] and L1 [39], respectively, were compared for their overall synteny and relationship (Additional file 9). These plasmids each contain eight major open reading frames (ORFs - potentially encode a protein) designated ORF18. Although the plasmid sequences vary in size (7169$7966 \mathrm{bp})$, alignment of their amino acid sequences revealed a high degree of similarity and large conserved regions (Additional files 9). The pCpnKo and pCpnE1 plasmid sequences share $96.2 \%$ identity and are more closely related to pCpA1 ( 81.3 and $78.9 \%$ identity), pCpGP1 (81.6 and 79.1\% identity) and pCfe1 (77.5 and $75.1 \%$ identity) than with pMoPn (69.5 and $67.7 \%$ identity), pCTA, pJALI, pSW2 and pLVG440 (63.9-69.5\% identity) (Additional file 10). Overall, there were approximately 30 indels among the species; three of the longest indels were identified in ORF1 of pCpnE1 (deletion), $\mathrm{pCfe} 1$ (insertion) and $\mathrm{pSW} 2$ (deletion). A phylogenetic tree was inferred from multiple sequence alignment of the amino acid sequence (Figure 2). Three main branches (supported with a high bootstrap value) were evident: (1) C. pneumoniae LPCoLN and N16; (2) C. psittaci N352, C. felis $\mathrm{Fe} / \mathrm{C}-56$ and C. caviae GPIC; (3) C. muridarum and C. trachomatis isolates.

The bacteriophage is another strain-specific extrachromosomal element reported in chlamydial species. In C. pneumoniae, AR39 is the only human genome to have an extrachromosomal bacteriophage (4524 nt single-stranded DNA) [25], whereas the koala LPCoLN genome showed remnants of a phage. The first remnant of a phage in the koala LPCoLN genome was evident in
CPK_ORF00729, a 366 nt incomplete ORF that is presumably defective, sharing approximately $79 \%$ similarity (nt 301/382) to the partial-length of the human AR39 phage. CPK_ORF00729 also shares 97\% similarity (nt 324/333) to the Chp1 remnant (C. psittaci phage), which is present in the four C. pneumoniae human genomes. This suggests earlier integration of the phage genome in the C. pneumoniae genome (Geng MM, Schuhmacher A, Muehldorfer I, Bensch KW, Schaefer KP, Schneider S, Pohl T, Essig A, Marre R, Melchers K: The genome sequence of Chlamydia pneumoniae TW183 and comparison with other Chlamydia strains based on whole genome sequence analysis, submitted). The second koala LPCoLN phage remnant was a $445 \mathrm{nt}$ ORF, termed CPK_ORF00730, which appears to be 'intact', sharing approximately $77 \%$ similarity (nt 342/ $445)$ to the human AR39 phage. The koala LPCoLN phage remnants are positioned between hypothetical genes in the genome and there appear to be no further remnants of the phage within this region of the genome. A comparison of the C. pneumoniae koala LPCoLN phage with other chlamydial species revealed approximately $77-79 \%$ sequence identity to partial-length sequences of the C. psittaci Chp2 phage, C. pecorum phage 3, C. caviae phiCPG1 phage and C. abortus Chp4 phage.

\section{Discussion}

In this work, we applied computational analyses to explore the genome content and genetic diversity among the recently sequenced $C$. pneumoniae koala LPCoLN genome and previously published C. pneumoniae human genomes (AR39, CWL029, J138 and TW183). The koala LPCoLN genome is larger than all four C. pneumoniae human genomes by $10-12 \mathrm{kbp}$. We combined BLAST search methods and motif analysis for use in elucidating the relationship between gene

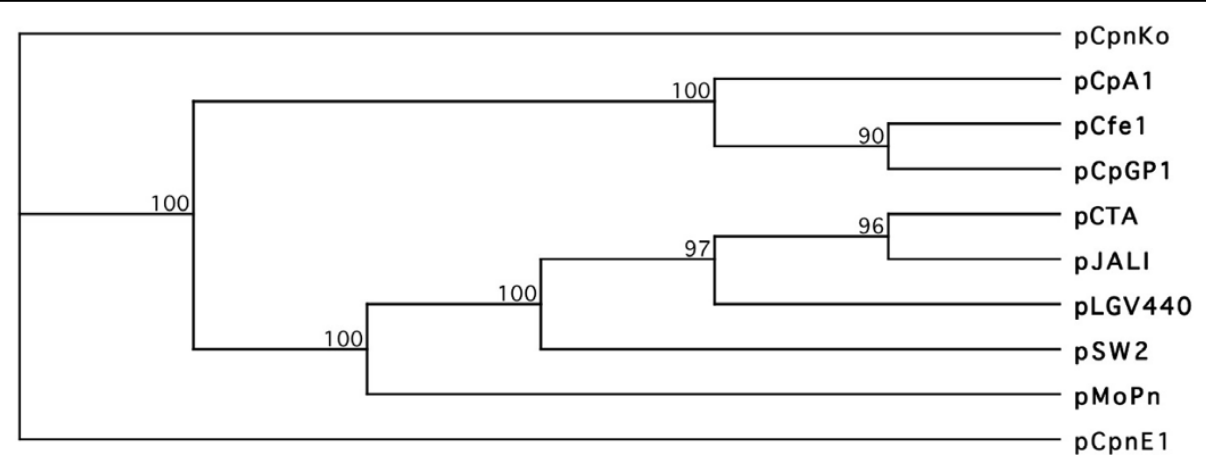

Figure 2 Phylogeny of the chlamydial plasmid. Phylogenetic relationships of C. pneumoniae koala LPCoLN (pCpnKo), C. pneumoniae horse N16 (pCpnE1), C. psittaci avian N352 (pCpA1), C. felis feline Fe/C-56 (pCfe1), C. caviae guinea pig GPIC (pCpGP1), C. muridarum mouse Nigg (pMoPn) and C. trachomatis human serovars A (pCTA), B (pJALI), E (pSW2) and L1 (pLVG440) were inferred from predicted amino acid sequences, and were constructed by Neighbor-Joining analysis and 1,000 bootstrap replicates. 
function and evolution. Even though these techniques have several limitations [40], our comparative approach has (i) identified genome plasticity, (ii) provided circumstantial evidence for the presumed direction of C. pneumoniae evolution, and (iii) suggested targets for detection and differentiation of C. pneumoniae isolates from both human and animal origins.

The presence of unique insertions/deletions is evidence of evolution in action. For the majority of these genes, the C. pneumoniae koala genome has the fulllength version. These length polymorphisms suggest that the presumed functional changes are brought about by adaptation to a specialised niche, where the ancestral gene function may no longer be required. Therefore, it is important to understand how these genetic differences may influence differences in pathogenicity and fitness in the host. Our data supports the findings of Rattei et al. [41] and the whole genome findings of Myers et al. [24] in that the essentially clonal human isolates have evolved from an animal strain(s) that has adapted to humans through fragmentation, decay and loss-of-function processes whereby the activity of the gene product may be reduced or specialised. Hence, the koala LPCoLN genome seems to be an 'older' strain in this sense. In addition, we provide new information on strain diversity and have identified targets for detection and further investigation.

Our analysis revealed a total of 140 genes that were specific to C. pneumoniae. One hundred and twentythree of these represented hypothetical genes with no significant similarity to genes in other organisms present in the database. Further analysis of these hypothetical genes (subcellular localisation, gene expression analysis, functional profiling from microarray analysis) may reveal undiscovered biovars or subspecies in C. pneumoniae.

The Pmp family is characterised by an unusual degree of sequence polymorphism, including mutations and large indels across all species [42-47] and showed variation within C. penumoniae. This suggests that the $p m p$ gene family is subjected to high selective pressure (niche, host-specific or immune-mediated), correlating with a relatively faster evolutionary rate for these antigens. Taken together, the polymorphism of $p m p$ sequences in C. pneumoniae from humans and animals is dually consistent with the divergent evolution of the pmp genes under host-specific selection while maintaining the capacity to adapt to specific niches or immune responses in the two different hosts.

In light of the variation seen between other families of genes and their orthologs in the human isolates, namely the pmp protein family, the strict conservation of T3S effector genes was initially surprising given the effectors' normal tendency for divergence. While the differences observed between other regions of the genomes are consistent with evolutionary changes [24], the relative conservation of effector genes over equivalent time suggest that changes in genes encoding effector proteins were likely selected against. This is consistent with a key role of T3S effectors in mediating steps of the biology of C. pneumoniae that are conserved in human and animal strains, such as inclusion and intracellular development. Overall, these results support a critical role for T3S less in the virulence than in the developmental biology of these organisms. Such a role has been proposed in the context of the contact-dependent T3S-mediated hypothesis of chlamydial development proposed earlier $[48,49]$.

Orthologs of the MACPF were identified in several chlamydial species. The first biological characterisation of the C. trachomatis MACPF by Taylor et al. [50] has revealed that the MACPF (CT153) might be activated by proteolytic processing and may play a role in the acquisition or modification of host-derived lipids. By contrast, studies of the MACPF in other organisms, including that of Toxoplasma spp. have shown that ablation of the MACPF (termed TgPLP1) resulted in a reduction in virulence (in mice), whereby TgPLP1 deficient parasites were unable to exit normally and were entrapped within host cells, due to the inability to permeabilise the parasitophorous vacuole membrane [51]. If the chlamydial MACPF was to play a similar role in egression or virulence, then why have several species failed to retain this gene? Non-lytic family members have also been identified in other organisms including Astrotactin involved in neural migration in mammals [52], a Drosophila torso-like protein involved in embryonic development [53] and Plu-MACPF of Photorhabdus luminescens which binds to the surface of insect cells [54]. Further investigation of this gene should provide more insight into its role in Chlamydiaceae.

C. pneumoniae is the only chlamydial species thus far to have a $u d k$ gene encoding uridine kinase. The $u d k$ gene is a pyrimidine ribonucleoside kinase that phosphorylates uridine and cytidine into uridine or cytidine monophosphate (UMP/CMP) [55], and is highly conserved in the species. It has been reported that the Prevotella bryantii genome encodes a putative uracil DNA glycosylase and uridine kinase, likely to be involved in the removal of misincorporated uracil from DNA and its subsequent re-use [56]. All chlamydial genomes also encode a uracil DNA glycosylase, however, C. pneumoniae is the only species carrying the $u d k$ ortholog. This implies that an alternative gene product is involved in UMP production in the other chlamydial species. The C. muridarum genome includes a CDS (upp) encoding a uracil phosphoribosyltransferase [25] that may represent the main pathway for UMP production in this species. C. pneumoniae is unusual in having a very broad host 
range and therefore the fact that it is the only chlamydial species to have retained the $u d k$ gene could reflect this broad host capacity.

Most bacteria can salvage or synthesise their own purines and pyrimidines. By contrast, chlamydiae and rickettsiae (another obligate intracellular bacterium) are incapable of de novo synthesis, and to a degree, of salvage [31,57]. Given the absence of genes for enzymes upstream in the pyrimidine biosynthesis pathway, it is unclear why the pyrE should be retained. The final step in the pathway is via $p y r F$ which is absent from the chlamydial genome, suggesting that they are unable to convert orotate to UMP. The presence in all chlamydial genomes of orthologs encoding the three downstream enzymes involved in UMP to CTP conversion and the earlier demonstration of CTP synthetase activity in these organisms $[58,59]$ confirm that chlamydiae are not auxotrophic for CTP. Furthermore, a three-gene cluster including guaB, guaA and add have been selectively maintained in several chlamydial species including C. pneumoniae AR39, CWL029, TW183 and J138, C. felis Fe/C-56, C. caviae GPIC and C. muridarum Nigg. C. abortus has a guaB pseudogene, whereas the C. pneumoniae LPCoLN and C. trachomatis serovar A/HAR, B/Jali20/OT, D/UQ-3/ $\mathrm{CX}, \mathrm{L} 2 \mathrm{~b} / \mathrm{UCH}-1 /$ proctititis, $\mathrm{L} 2 / 434 / \mathrm{Bu}$ and Candidatus Protochlamydia amoebophila UWE25 genomes lack all three genes $[25,26,30,35,36,60]$. The selective loss of guaBA-add from C. pneumoniae koala LPCoLN and other chlamydial species suggest that these three enzymes required for inter-conversion of GMP, IMP and AMP must be acquired by other means or are clearly not essential for species survival.

Copy number variations of the tyrP (tryptophan tyrosine permease) gene have been suggested to reflect vascular tropism and pathogenicity among C. pneumoniae human isolates with multiple copies associated with respiratory infection and single copy more frequently associated with vascular tropism [33]. A comparison of the five sequenced $C$. pneumoniae genomes also revealed variations in the tyrP (tryptophan tyrosine permease) copy number that are, however, inconsistent with the hypothesis by Gieffers et al. [33]. Among these, two respiratory isolates (koala LPCoLN and human J138) $[24,30]$, as well as the single conjunctival isolate (TW183) of the group (Geng MM, Schuhmacher A, Muehldorfer I, Bensch KW, Schaefer KP, Schneider S, Pohl T, Essig A, Marre R, Melchers K: The genome sequence of Chlamydia pneumoniae TW183 and comparison with other Chlamydia strains based on whole genome sequence analysis, submitted) have a single tyr $\mathrm{P}$ copy while two other respiratory isolates (CWL029 and AR39) $[25,26]$ carry duplicate copies of tyrP.
The loss and fragmentation of pre-existing genes during evolution is one of the primary distinguishing features between C. pneumoniae koala and C. pneumoniae human. Extrachromosomal plasmids have been identified in six of the nine chlamydial species. As the plasmid is not common to C. pneumoniae, it is not known why koala LPCoLN has a plasmid.

While proteins with predicted or known biologic function are favoured candidate gene targets, many C. pneumoniae-specific hypothetical proteins with no predicted function were identified in the comparisons and may be worth further investigating for their potential role in host tropism, pathogenicity and niche adaptation (see Additional file 2 for the list of genes). A suggested list of target genes for C. pneumoniae detection and a brief description of their characteristics is summarised in additional file 11. Selected genes include (i) C. pneumoniae-specific genes for detection of C. pneumoniae, (ii) genes that could potentially differentiate isolates from human and animal origins, for example, length polymorphic genes including the membrane attack complex perforin and the hypothetical protein CPK_ORF00679, (iii) genes for the identification of a C. pneumoniae plasmid.

\section{Conclusions}

The study of whole genome sequences provides important clues to the natural history of C. pneumoniae and their hosts, and assists in the identification of functional differences that may determine pathogenicity and virulence differences between the strains. Previously, whole genome comparisons have focused on four practically clonal C. pneumoniae human genomes, which make the identification of target genes for strain differentiation quite challenging. In this study we have made use of the recently sequenced koala LPCoLN genome, which is the largest and most unique C. pneumoniae genome sequenced thus far, in order to select target genes that represented traits of strain-specific determinants. Further investigation of these genes in other host species may provide additional clues as to what enables this pathogen to: (i) present varied clinical pathologies, (ii) occupy multiple niches, and (iii) establish an infection in cold and warm-blooded hosts. Moreover, these genes may become targets for improved diagnosis and therapeutic strategies.

\section{Methods}

\section{Chlamydia pneumoniae isolates}

The complete genomic sequences of C. pneumoniae AR39 (GenBank accession number AE002161), CWL029 (GenBank accession number AE001363) TW183 (GenBank accession number AE009440), J138 (GenBank 
accession number BA000008) and LPCoLN (GenBank accession CP001713) were used in this study.

\section{Strategy for selection of genes used for comparisons}

The selection strategy involved individual gene-by-gene comparisons of the complete koala LPCoLN genome. NCBI BLAST [61] searches using tblastx and tblastn were conducted in order to search for orthologs in the C. pneumoniae human genome and other organisms using an E-value cutoff of $1 \times 10^{-4}$ with manual curation. This approach enabled us to identify regions of high SNP accumulation and to select target genes for comparison. These selected genes were grouped into five categories by their putative, predicted or hypothetical functions. The categories included: (1) C. pneumoniae-specific genes (with respect to the koala LPCoLN genome, $\mathrm{n}=140$ ); (2) genes with a prior demonstrated role in chlamydial biology and pathogenicity $(n=49)$; (3) genes encoding nucleotide salvage or amino acid biosynthesis proteins $(\mathrm{n}=6)$; (4) extrachromosomal elements, including a plasmid $(\mathrm{n}=9)$ and bacteriophage-related genes $(n=2)$ (Additional files 1 and 2). Selected genes were individually aligned in order to identify SNPs, indels and targets for strainspecific adaptations.

\section{Analysis of nucleotide and amino acid sequences and phylogeny}

Gene alignments were performed using the Clustal W program (MacVector 10.6 Genetics Computer Group, Madison, Wisconcin) to identify polymorphisms and indels (insertions/deletions) [62]. Conserved residues are outlined, similar amino acids are shaded in grey, mismatches are not shaded and dashes correspond to gaps in the sequence. A consensus line appears at the bottom of the alignment. Pmp sequences were subjected to multiple sequence alignment using ClustalW2 (EMBL-EBI) [63] and BioEdit Sequence Alignment Editor [64]. SNP position analysis for each group was performed using the Microsoft Excel program (Microsoft Corporation). Motif Scan [65] was used to identify motifs in a sequence.

A phylogenetic tree was constructed by NeighborJoining, tie breaking = systematic, distance corrected by the Poisson method with gaps distributed proportionally and 1,000 bootstrap replicates.

\section{Additional material}

Additional file 1: Categories of targets for investigation. (1) gene with a prior demonstrated role in chlamydial biology and pathogenicity $(n=49)$; (2) genes encoding nucleotide salvage or amino acid biosynthesis proteins $(n=6)$; (3) extrachromosomal elements, including a plasmid $(n=9)$ and bacteriophage-related genes $(n=2)$.
Additional file 2: C. pneumoniae-specific genes. A list of $C$. pneumoniae genes that have no significant similarity to other chlamydial species or organisms. E-value cutoff of $1 \times 10^{-4}$ with manual curation.

Additional file 3: Comparative analysis of the $C$. pneumoniae polymorphic membrane proteins (Pmps). A comparison of the 21 Pmps revealed a high degree of sequence polymorphism and indels between the koala LPCoLN and human AR39, CWL029 and TW183 isolates. The TW183 and J138 isolates were well-conserved and therefore, TW183 also represents $\mathrm{J} 138$ in this figure. Arrows indicate the direction of transcription: green arrows show typical $p m p$ characteristics, red arrows represent pseudogenes (numbers below arrows indicate stop codon position), and white arrows represent proteins with no relation to pmps (note the same orientation as pmpG10). Dashed lines indicate truncated products.

Additional file 4: Polymorphic outer membrane protein features of C. pneumoniae

Additional file 5: Polymorphic outer membrane protein SNP position analysis of $C$. pneumoniae.

Additional file 6: T3S ortholog comparisons.

Additional file 7: Chlamydia MACPF. A BLAST alignment of the C. pneumoniae MACPF protein. From top to bottom: C. pneumoniae LPCoLN, C. pneumoniae J138, C. pneumoniae CWL029, C. felis FE/C-56, C. trachomatis A/HAR-13, C. trachomatis 6276, C. trachomatis D/UW-3/CX, C. trachomatis 70, C. trachomatis 434/Bu, C. muridarum Nigg, C. pneumoniae CWL029, C. abortus S26/3, C. felis Fe/C-56, Alcanivorax sp DG881, Saccolglossus kowaleski, Theileria parva strain Muguga....

Additional file 8: Chlamydia has lost several steps in the pyrimidine biosynthesis pathway. All chlamydial genomes sequenced thus far, have lost the initial steps involved in pyrimidine biosynthesis. C. pneumoniae (Cpn), C. abortus (Cab), C. caviae (Cav) and C. felis (Cfe) contain a pyrE gene encoding an orotate phosphoribosyltransferase, while C. muridarum (Cmu) and C. trachomatis (Ctr) lack this gene. The next step in the pathway is via pyrF, which is absent from all chlamydial genomes. Interestingly, all six genomes have maintained the last three steps for the conversion of UMP into CTP. Adapted from Koonin and Galperin [66]. Gene names: carA, carbamoyl-phosphate synthase, small subunit; carB, carbamoyl-phosphate synthase, large subunit; pyrB, aspartate carbamoyltransferase; $p y r C$, dihydroorotase; $p y r D$, dihydroorotate dehydrogenase; $p y r E$, orotate phosphoribosyltransferase; $p y r F$, orotidine 5-phosphate decarboxylase; $p y r H$, uridylate kinase; $n d k$, nucleoside diphosphate kinase; pyrG, CTP synthase. Red boxes indicate gene loss, yellow boxes indicate the presence of a gene.

Additional file 9: Sequence comparison of the chlamydial plasmid. Multiple sequence alignment of the predicted amino acid sequence from C. pneumoniae koala LPCoLN (pCpnKo), C. pneumoniae horse N16 (pCpnE1), C. psittaci avian N352 (pCpA1), C. felis feline Fe/C-56 (pCfe1), C. caviae guinea pig GPIC (pCpGP1), C. muridarum mouse Nigg (pMoPn) and $C$. trachomatis human serovars $A(p C T A), B(p J A L I), E(p S W 2)$ and L1 ( $p L V G 440$ ). The sequences are well-conserved across species, indicating some degree of ancestry among them. The C. pneumoniae plasmid shared a close relationship with C. psittaci, C. caviae and C. felis, while C. muridarum and C. trachomatis were highly conserved. Predicted functions include plasmid replication (ORF1 and ORF2), double-stranded DNA unwinding (ORF3), chlamydial pathogenesis (ORF5) and regulation of partitioning and copy number (ORF7 and ORF8) [34,67]. The functions of ORF4 and ORF6 remain to be determined.

Additional file 10: Plasmid similarity scores (\%). Plasmid similarity scores based on multiple sequence alignment.

Additional file 11: List of $C$. pneumoniae target genes. Suggested target genes for detection, strain differentiation and plasmid identification in C. pneumoniae. See also reference [68].

\section{Abbreviations}

CDS: Coding Sequence; Indels: Insertions/Deletions; ORF: Open Reading Frame; SNP: Single Nucleotide Polymorphism. 


\section{Acknowledgements}

This work was supported by the National Institute of Allergy and Infectious Disease grant 1R01AI051472. PMB and JAC were supported by NIH/NIAID research grant RO1 Al51417. KH was supported by $\mathrm{NIH/NIDCR}$ training grant T32 DE07309.

\section{Author details}

'Institute of Health and Biomedical Innovation, Faculty of Science and Technology, Queensland University of Technology, Kelvin Grove, Queensland, 4059, Australia. ${ }^{2}$ Department of Microbial Pathogenesis, University of Maryland, Baltimore, Maryland 21201, USA. ${ }^{3}$ Institute for Genome Sciences, University of Maryland, Baltimore, Maryland 21201, USA.

\section{Authors' contributions}

CMM prepared the manuscript and performed comparative analyses including evaluations. KMH contributed with text, interpretation and analysis for the manuscript. PMB aided in data interpretation and critically revised the manuscript. GSAM interpretation and analysis of the genome. JAC contributed with text, interpretation and analysis for the manuscript. PT participated in the design and drafting of the manuscript, aided in data interpretation and critically revised the manuscript. All authors read and approved the final manuscript.

Received: 6 November 2009 Accepted: 21 July 2010

Published: 21 July 2010

\section{References}

1. Grayston JT: Immunisation against trachoma. Pan Am Health organ Sci Publ 1965, 147:549.

2. Storey CC, Lusher M, Yates P, Richmond SJ: Evidence for Chlamydia pneumoniae of non-human origin. J Gen Microbiol 1993, 139:2621-2626.

3. Berger L, Volp K, Mathews S, Speare R, Timms P: Chlamydia pneumoniae in a free-ranging giant barred frog (Mixophyes iteratus) from Australia. J Clin Microbiol 1999, 37:2378-2380.

4. Hotzel H, Grossmann E, Mutschmann F, Sachse K: Genetic characterization of a Chlamydophila pneumoniae isolate from an African frog and comparison to currently accepted biovars. Syst Appl Microbiol 2001, 24:63-66.

5. Bodetti TJ, Jacobson E, Wan C, Hafner L, Pospischil A, Rose K, Timms P: Molecular evidence to support the expansion of the hostrange of Chlamydophila pneumoniae to include reptiles as well as humans, horses, koalas and amphibians. Syst App Microbiol 2002, 25:146-152.

6. Saikku P, Leinonen M, Mattila K, Ekman MR, Nieminen MS, Makela PH, Huttunen JK, Valtonen V: Serological evidence of an association of a novel Chlamydia, TWAR, with chronic coronary heart disease and acute myocardial infarction. Lancet 1988, 2:983-986.

7. Grayston JT: Background and current knowledge of Chlamydia pneumoniae and atherosclerosis. J Infect Dis 2000, 181(Suppl 3):S402-S410.

8. Wesslen L, Pahlson C, Friman G, Fohlman J, Lindquist O, Johansson C: Myocarditis caused by Chlamydia pneumoniae (TWAR) and sudden unexpected death in a Swedish elite orienteer. Lancet 1992, 340:427-428.

9. Sriram S, Mitchell W, Stratton C: Multiple sclerosis associated with Chlamydia pneumoniae infection of the CNS. Neurology 1998, 50:571-572.

10. Balin BJ, Gerard HC, Arking EJ, Appelt DM, Branigan PJ, Abrams JT, Whittum-Hudson JA, Huson AP: Identification and localization of Chlamydia pneumoniae in the Alzheimer's brain. Med Microbiol Immunol 1998, 187:23-42.

11. Girjes AA, Huggal AF, Timms P, Lavin MF: Two distinct forms of Chlamydia psittaci associated with disease and infertility in Phascolarctos cinereus (koala). Infect Immun 1988, 56:1897-1900.

12. Girjes AA, Hugall A, Graham DM, MCCaul TF, Lavin MF: Comparison of type I and type II Chlamydia psittaci strains infecting koalas (Phascolarctos cinereus). Vet Microbiol 1993, 37:65-83.

13. Jackson M, White N, Giffard P, Timms P: Epizootiology of Chlamydia infections in two free-range koala populations. Vet Microbial 1999, 65:255-264.

14. Devereaux LN, Polkinghorne A, Meijer A, Timms P: Molecular evidence for novel chlamydial infections in the koala (Phascolarctos cinereus). Syst Appl Microbiol 2003, 26:245-253.
15. McColl KA, Martin RW, Gleeson LH, Handasyde KA, Lee AK: Chlamydia infection and infertility in the female koala (Phascolarctos cinereus). Vet Rec 1984, 115:655.

16. Brown AS, Girjes AA, Lavin MF, Timms P, Woolcock JB: Chlamydial disease in koalas. Aust Vet J 1987, 64:346-349.

17. Mitchell CM, Mathews SA, Theodoropoulos C, Timms P: In vitro characterisation of koala Chlamydia pneumoniae: morphology, inclusion development and doubling time. Vet Microbiol 2009, 136:91-99.

18. Yamazaki T, Nakada H, Sakurai N, Kuo CC, Wang SP, Grayston JT: Transmission of Chlamydia pneumoniae in young children in a Japanese family. J Infect Dis 1990, 162:1390-1392.

19. Mordhorst CH, Wang SP, Grayston JT: Outbreak of Chlamydia pneumoniae infection in four farm families. Eur J Clin Microbiol Infect Dis 1992, 11:617-620.

20. Troy CJ, Peeling RW, Ellis AG, Hockin JC, Bennett DA, Murphy MR, Spika JS: Chlamydia pneumoniae as a new source of infectious outbreaks in nursing homes. JAMA 1997, 277:1214-1218.

21. Kleemola M, Saikku P, Visakorpi R, Wang SP, Grayston JT: Epidemics of pneumonia caused by TWAR, a new Chlamydia organism, in military trainees in Finland. J Infect Dis 1988, 157:230-236.

22. Wills JM, Watson G, Lusher M, Mair TS, Wood D, Richmond SJ: Characterisation of Chlamydia psittaci isolated from a horse. Vet Microbiol 1990, 24:11-19.

23. Reed KD, Ruth GR, Meyer JA, Shukla SK: Chlamydia pneumoniae infection in a breeding colony of African clawed frogs (Xenopus tropicalis). Emerg Infect Dis 2000, 6:196-199.

24. Myers GSA, Mathews SA, Eppinger M, Mitchell C, O'Brien KK, White OR, Benahmed F, Brunham RC, Read TD, Ravel J, Bavoil PM, Timms P: Evidence that human Chlamydia pneumoniae was zoonotically acquired. J Bacteriol 191:7225-7233.

25. Read T, Brunham R, Shen C, Gill S, Heidelberg J, White O, Hickey E, Peterson J, Utterback T, Berry K, Bass S, Linher K, Weidman J, Khouri H, Craven B, Bowman C, Dodson R, Gwinn M, Nelson W, DeBoy R, Kolonay J, McClarty G, Salzberg S, Eisen J, Fraser C: Genome sequences of Chlamydia trachomatis MoPn and Chlamydia pneumoniae AR39. Nucleic Acids Res 2000, 28:1397-1406.

26. Kalman S, Mitchell W, Marathe R, Lammel C, Fan J, Hyman RW, Olinger L, Grimwood J, Davis RW, Stephens RS: Comparative genomes of Chlamydia pneumoniae and C. trachomatis. Nat Genet 1999, 4:385-389.

27. Cevenini R, Donati M, Brocchi E, De Simone F, La Placa M: Partial characterization of an $89-\mathrm{kDa}$ highly immunoreactive protein from Chlamydia psittaci A/22 causing ovine abortion. FEMS Microbiol Lett 1991, 65:111-115.

28. Longbottom D, Findlay J, Vretou E, Dunbar SM: Immunoelectron microscopic localisation of the OMP90 family on the outer membrane surface of Chlamydia psittaci. FEMS Microbiol Lett 1998, 164:111-117.

29. Stephens RS, Kalman S, Lammel C, Fan J, Marathe R, Aravind L, Mitchell W, Olinger L, Tatusov RL, Zhao Q, Koonin EV, Davis RW: Genome sequence of an obligate intracellular pathogen of humans: Chlamydia trachomatis. Science 1998, 282:754-759.

30. Shirai M, Hirakawa H, Kimoto M, Tabuchi M, Kishi F, Ouchi K, Shiba T, Ishii K, Hattori M, Kuhara S, Nakazawa T: Comparison of whole genome sequences of Chlamydia pneumoniae J138 from Japan and CWL029 from USA. Nucleic Acids Res 2000, 28:2311-2314.

31. Tipples G, McClarty G: The obligate intracellular bacterium Chlamydia trachomatis is auxotrophic for three of the four ribonucleoside triphosphates. Mol Microbiol 1993, 8:1105-1114.

32. Abromaitis S, Hefty PS, Stephens RS: Chlamydia pneumoniae encodes a functional aromatic amino acid hydroxylase. FEMS Immunol Med Microbiol 2009, 55:196-205.

33. Gieffers J, Durling L, Ouellette SP, Rupp J, Maass M, Byrne Gl, Caldwell HD, Belland RJ: Genotypic differences in the Chlamydia pneumoniae tyrP locus related to vascular tropism and pathogenicity. J Infect Dis 2003, 188:1085-1093.

34. Thomas NS, Lusher M, Storey CC, Clarke IN: Plasmid diversity in Chlamydia. Microbiology 1997, 143:1847-1854.

35. Azuma Y, Hirakawa H, Yamashita A, Cai Y, Rahman MA, Suzuki H, Mitaku S, Toh H, Goto S, Murakami T, Sugi K, Hayashi H, Fukushi H, Hattori M, Kuhara S, Shirai M: Genome sequence of the cat pathogen, Chlamydophila felis. DNA Res 2006, 13:15-23. 
36. Read TD, Myers GS, Brunham RC, Nelson WC, Paulsen IT, Heidelberg J, Holtzapple E, Khouri H, Federova NB, Carty HA, Umayam LA, Haft DH, Peterson J, Beanan MJ, White O, Salzberg SL, Hsia RC, McClarty G, Rank RG, Bavoil PM, Fraser CM: Genome sequence of Chlamydophila caviae (Chlamydia psittaci GPIC): examining the role of niche-specific genes in the evolution of the Chlamydiaceae. Nucleic Acids Res 2003, 31:2134-2147.

37. Carlson JH, Porcella SF, McClarty G, Caldwell HD: Comparative genomic analysis of Chlamydia trachomatis oculotropic and genitotropic strains. Infection and Immunity 2005, 73:6407-6418.

38. Seth-Smith HM, Harris SR, Persson K, Marsh P, Barron A, Bignell A, Bjartling C, Clark L, Cutcliffe LT, Lambden PR, Lennard N, Lockey SJ, Quail MA, Salim O, Skilton RJ, Wang Y, Holland MJ, Parkhill J, Thomson NR, Clarke IN: Co-evolution of genomes and plasmids within Chlamydia trachomatis and the emergence in Sweden of a new variant strain. BMC Genomics 2009, 10:239.

39. Hatt C, Ward ME, Clarke IN: Analysis of the entire nucleotide sequence of the cryptic plasmid of Chlamydia trachomatis serovar L1. Evidence for involvement in DNA replication. Nucleic Acids Res 1988, 16:4053-4067.

40. Gupta RS, Griffiths E: Chlamydiae-specific proteins and indels: novel tools for studies. Trends Microbiol 2006, 14:527-535.

41. Rattei T, Ott S, Gutacker M, Rupp J, Maass M, Shreiber S, Solbach W, Wirth T, Gieffers J: Genetic diversity of the obligate intracellular bacterium Chlamydophila pneumoniae by genome-wide analysis of single nucleotide polymorphisms: evidence for highly clonal population structure. BMC Genomics 2007, 8:355.

42. Grimwood J, Stephens RS: Computational analysis of the polymorphic membrane protein superfamily of Chlamydia trachomatis and Chlamydia pneumoniae. Microb Comp Genomics 1999, 4:187-201.

43. Gomes JP, Bruno WJ, Borrego MJ, Dean D: Recombination in the genome of Chlamydia trachomatis involving the polymorphic membrane protein C gene relative to ompA and evidence for horizontal gene transfer. $J$ Bacteriol 2004, 186:4295-4306.

44. Gomes JP, Nunes A, Bruno WJ, Borrego MJ, Florindo C, Dean D: Polymorphisms in the nine polymorphic, membrane proteins of Chlamydia trachomatis across all serovars: evidence for serovar Da recombination and correlation with tissue tropism. J Bacteriol 2006, 188:275-286.

45. Shirai M, Hirakawa H, Ouchi K, Tabuchi M, Kishi F, Kimoto M, Takeuchi H, Nishida J, Shibata K, Fujinaga R, Yoneda H, Matsushima H, Tanaka C, Furukawa S, Miura K, Nakazawa A, Ishii K, Shiba T, Hattori M, Kuhara S, Nakazawa T: Comparison of outer membrane protein genes omp and pmp in the whole genome sequences of Chlamydia pneumoniae isolates from Japan and the United States. J Infect Dis 2000, 181(Suppl 3): S524-S527.

46. Rocha EP, Pradillon O, Bui H, Sayada C, Denamur E: A new family of highly variable proteins in the Chlamydophila pneumoniae genome. Nucleic Acids Res 2002, 30:4351-4360.

47. Grimwood J, Olinger L, Stephens RS: Expression of Chlamydia pneumoniae polymorphic membrane protein family genes. Infect Immun 2001 69:2383-2389.

48. Wilson DP, Timms P, McElwain DLS, Bavoil PM: Type III secretion, contactdependent model for the intracellular development of Chlamydia. Bull Math Biol 2006, 68:161-178.

49. Peters J, Wilson DP, Myers G, Timms P, Bavoil PM: Type III secretion a la Chlamydia. Trends Microbiol 2007, 15:241-251.

50. Taylor LD, Nelson DE, Dorward DW, Whitmire WM, Caldwell HD: Biological characterization of Chlamydia trachomatis Plasticity Zone MACPF domain family protein CT153. Infect Immun 2010, 76:2691-2699.

51. Kafsack BF, Pena JD, Coppens I, Ravindran S, Boothroyd JC, Carruthers VB: Rapid membrane disruption by a perforin-like protein facilitates parasite exit from host cells. Science 2009, 323:530-533.

52. Zheng C, Heintz N, Hatten ME: CNS gene encoding astrotactin, which supports neuronal migration along glial fibers. Science 1996, 272:417-419.

53. Martin JR, Raibaud A, Ollo R: Terminal pattern elements in Drosophila embryo induced by the torso-like protein. Nature 1994, 367:741-745.

54. Rosado CJ, Buckle AM, Law RHP, Butcher RE, Kan W-T, Bird CH, Ung K, Browne KA, Baran K, Bashtannyk-Puhalovich TA, Faux NG, Wong W, Porter CJ, Pike RN, Ellisdon AM, Pearce MC, Bottomley SP, Emsley J, Smith Al, Rossjohn J, Hartland EL, Voskoboinik I, Trapani JA, Bird PI, Dunstone MA, Whisstock JC: A common fold mediates vertebrate defense and bacterial attack. Science 2007, 317:1548-1551.
55. Martinussen J, Hammer K: Powerful methods to establish chromosomal markers in Lactococcus lactis: an analysis of pyrimidine salvage pathway mutants obtained by positive selections. Microbiol 1995, 141:1883-1890.

56. Accetto T, Avgustin G: Expression of nuclease gene nucA, a member of an operon putatively involved in uracil removal from DNA and its subsequent reuse in Prevotella bryantii. Arch Microbiol 2008, 190:111-117.

57. Winkler H: Rickettsia species (as organisms). Annu Rev Microbiol 1990, 44:131-153.

58. Hatch TP: Utilization of L-cell nucleoside triphosphates by Chlamydia psittaci for ribonucleic acid synthesis. J Bacteriol 1975, 122:393-400.

59. McClarty G, Tipples G: In situ studies on incorporation of nucleic acid precursors into Chlamydia trachomatis DNA. J Bacteriol 1991, 173:4922-4931.

60. Thomson NR, Yeats C, Bell K, Holden MTG, Bentley SD, Livingstone M, Cerdeno-Tarraga AM, Harris B, Doggett J, Ormond D, Mungall K, Clarke K, Feltwell T, Hance Z, Sanders M, Quail MA, Price C, Barrell BG, Parkhill J, Longbottom D: The Chlamydophila abortus genome sequence reveals an array of variable proteins that contribute to interspecies variation. Genome Research 2005, 15:629-640.

61. NCBI (National Centre for Biotechnology Information) BLAST (Basic Local Alignment Search Tool). [http://blast.ncbi.nlm.nih.gov/Blast.cgi].

62. Thompson JD, Higgins DG, Gibson TJ: CLUSTAL W: improving the sensitivity of progressive multiple sequence alignment through sequence weighting, position-specific gap penalties and weight matrix choice. Nucleic Acids Res 1994, 22:4673-4680.

63. Larkin MA, Blackshields G, Brown NP, Chenna R, McGettigan PA, McWilliam H, Valentin F, Wallace IM, Wilm A, Lopez R, Thompson JD, Gibson TJ, Higgins DG: Clustal W and Clustal X version 2.0. Bioinformatics 2007, 23:2947-2948.

64. Hall TA: BioEdit: a user-friendly biological sequence alignment editor and analysis program for Windows 95/98/NT. Nucl Acids Symp Ser 1999, 41:95-98.

65. My hits, motif scan. [http://myhits.isb-sib.ch/cgi-bin/motif_scan].

66. Koonin EV, Galperin MY: Sequence-evolution-function: Computational approaches in comparative genomics. Norwell, Massachusetts: Kluwer Academic Publishers 2003, 39.

67. Li Z, Chen D, Zhong Y, Wang S, Zhong G: The chlamydial plasmidencoded protein pgp3 is secreted into the cytosol of Chlamydia-infected cells. Infect Immun 2008, 76:3415-3428.

68. Mitchell CM, Hutton S, Myers GSA, Brunham R, Timms P: Chlamydia pneumoniae is genetically diverse in animals and appears to have crossed the host barrier to humans on (at least) two occasions. Plos Path

doi:10.1186/1471-2164-11-442

Cite this article as: Mitchell et al: Comparison of koala LPCoLN and human strains of Chlamydia pneumoniae highlights extended genetic diversity in the species. BMC Genomics 2010 11:442.

\section{Submit your next manuscript to BioMed Central and take full advantage of:}

- Convenient online submission

- Thorough peer review

- No space constraints or color figure charges

- Immediate publication on acceptance

- Inclusion in PubMed, CAS, Scopus and Google Scholar

- Research which is freely available for redistribution

Submit your manuscript at www.biomedcentral.com/submit
C BioMed Central 